PUPT-1923

\title{
A Note on Warped String Compactification
}

\author{
Chang S. Chan ${ }^{1}$, Percy L. Paul ${ }^{2}$ and Herman Verlinde ${ }^{1}$ \\ 1 Joseph Henry Laboratories, Princeton University, Princeton NJ 08544 \\ ${ }^{2}$ National Research Council, 100 Sussex Drive, Ottawa Ontario K1A OR6
}

\begin{abstract}
We give a short review of a large class of warped string geometries, obtained via F-theory compactified on Calabi-Yau fourfolds, that upon reduction to 5 dimensions give consistent supersymmetric realizations of the RS compactification scenario.
\end{abstract}




\section{Introduction}

This note is intended to clarify the realization and interpretation of the Randall-Sundrum compactification scenario within string theory. In the model of [1], our 4-d world is extended with an extra direction $r$ to a 5-d space-time with the warped metric

$$
d s^{2}=e^{2 \sigma(r)} \eta_{\mu \nu} d x^{\mu} d x^{\nu}+d r^{2}
$$

with $\sigma(r)=-k|r|$. Even while the range of $r$ is infinite, the warped form of the metric ensures that the effective volume of the extra direction is finite. As a result, matter particles sufficiently close to the domain wall region near $r=0$ will experience ordinary 4-d gravity at long distances [1].

At first sight, this proposal seems like a rather drastic departure from the more conventional Kaluza-Klein framework. Indeed, in most works on string compactifications thus far, the four uncompactified directions and the compact manifold are assumed to form a simple direct product. Although it was realized for a long time that this basic KK set-up can be generalized to include the possibility of warped products, the physics of these more general scenarios is still largely unexplored.

A second important ingredient of the RS-scenario is that part or all of the observable matter may be thought of as confined to a 4-d sub-manifold of the higher dimensional space-time. A concrete theoretical realization of such world-branes are the D3-branes of IIB string theory, which confine open strings to their world-volume. D3-branes, however, do not bind 4-d gravity. Possible supersymmetric realizations of the Planck-brane, located around $r=0$ in (1), are therefore rather expected to be found in the form of domain wall type configurations, or possible stringy generalizations thereof. Various attempts have been made to find smooth domain wall solutions of this type within 5-d gauged supergravity, but thus far without real success [2] [3].

There are several reasons for why this is indeed a hard problem. Even for a given compactification from 10 dimensions, it is an elaborate task to derive the dimensionally reduced theory. Thus far this has been done only for reductions over rather special symmetric 5-manifolds $K_{5}$ such as $S^{5}$ or $S^{5} / Z_{2}$, etc, and/or for special theories with extended supersymmetry. However, while it seems feasible to classify the possible types of supersymmetric solutions for each of these special dimensional reductions, there is no guarantee that they provide a general enough framework.

Instead of following the above procedure of (1) performing some special dimensional reduction to 5 dimensions and (2) looking for RS domain wall type solutions, it seems 


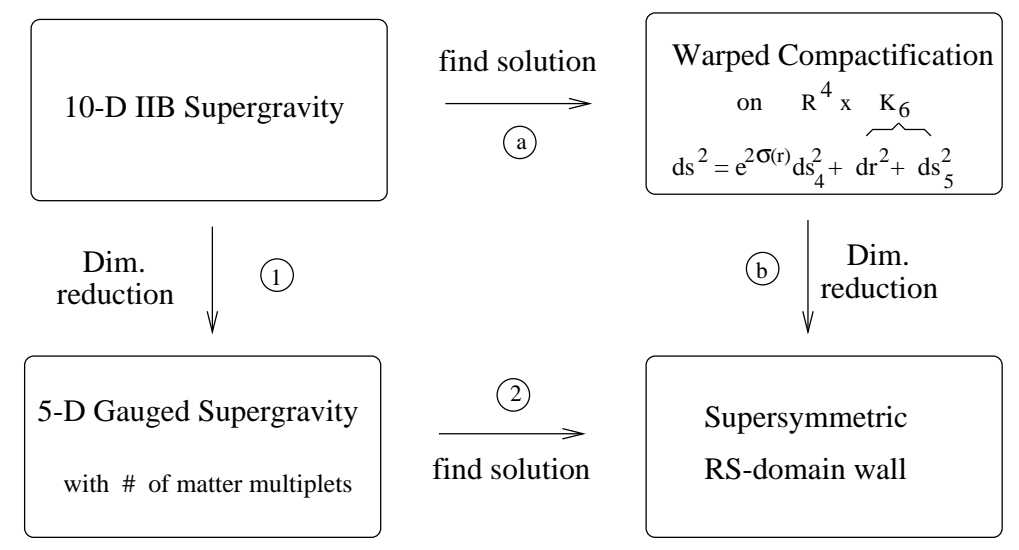

Fig 1. To identify supersymmetric RS-type geometries, we will follow the route IIB $->$ (a) $->$ (b) $->$ RS. It is still an open problem to find a direct construction of these geometries via the other route.

more practical to reverse the two steps. Since the scalar fields $\phi^{a}$ arise as moduli of some internal 5-d compact space $K_{5}$, any domain wall solution in 5-d gauged supergravity describes (upon lifting it back up to 10-dimensions) some specific warped compactification of the 10-d theory. It will therefore be much more general - and also easier - to first (a) identify a general class of warped compactifications of the 10-dimensional theory, and then (b) perform the same type of dimensional reduction from 10 to 5 dimensions. In the end, one can then hope to identify a class of 10-d geometries for which the resulting dimensionally reduced solution has all the required properties.

As will be described below, such a class of warped IIB geometries indeed exists in the form of quite generic F-theory compactifications on Calabi-Yau four-folds. If These have been studied in some detail in the recent literature - a list of references include [5] [6] [7] 8] and [9] - and indeed none of our equations will be new. Given the current interest in the subject, however, it seems useful to collect some of the known facts about these compactifications, since it has not been generally appreciated that supersymmetric RS-geometries indeed exist in string theory, and furthermore that they are in fact quite generic.

Since all derivations are contained in existing papers, we will here only present the general form of the compactification geometry without any proof that it is really a supersymmetric solution to the 10-d equations of motion. This proof can however be quite directly extracted from the literature, in particular from the very clear discussion by

\footnotetext{
${ }^{1}$ Another special realisation of an RS geometry in terms of a toroidal type IIB orientifold compactification has been described in [4].
} 
Becker and Becker [5]. Their analysis was done in the context of M-theory compactifications on $\mathrm{C}$-Y four-folds. It can however be straightforwardly translated to the F-theory context by performing the T-duality transformation outlined in [7]. An explicit example of this T-duality transformation is discussed in [6].

Although the 10-d perspective will allow us to identify a large class of RS-type compactification geometries, their geometrical structure is rather involved. It is therefore not easy to explicitly perform the dimensional reduction of these solutions to 5-dimensions. We will nonetheless attempt to make this 5 -d perspective as transparent as possible. In particular we will show that they indeed give rise to a 5 -d metric of the generic form (1).

\section{Warped Compactification in F-theory}

F-theory is a geometric language for describing compactifications of type IIB string theory, in which the expectation values of the dilaton and axion fields are allowed to vary nontrivially along the compactification manifold [10]. Compactifications of F-theory down to four-dimensions are specified by means of a Calabi-Yau four-fold that admit an elliptic fibration with a section. In other words, these are 8 dimensional compact manifolds $K_{8}$ that locally look like a product of a complex three-fold $K_{6}$ times a two-torus $T^{2}$. The two-torus will be taken to shrink to zero size. It can however be taken to change its shape when moving along the base $K_{6}$. In particular, it can have non-trivial monodromies around singular co-dimension 2 loci inside the $K_{6}$, where the elliptic fiber degenerates.

The four-fold $K_{8}$ is not the actual compactification geometry; rather it gives an economical way to characterize the compactification geometry as well as the expectation values of other fields. Moreover, due to the special geometric properties of the CalabiYau four-fold $K_{8}$ - vanishing first Chern class and $S U(4)$ holonomy - the associated IIB background by construction will preserve 4-d supersymmetry, at least at the classical and perturbative level.

The warped geometry of this type of F-theory compactifications has been derived in [6], by direct translation of the M-theory analysis of [5]. The full solution for the 10-dimensional IIB string metric, in the string frame, takes the form

$$
d s_{I I B}^{2}=e^{2 \alpha(y)} \eta_{\mu \nu} d x^{\mu} d x^{\nu}+e^{-2 \alpha(y)} g_{\mathrm{MN}}(y) d y^{\mathrm{M}} d y^{\mathrm{N}}
$$

where $g_{\mathrm{MN}}$ denotes the metric on the 6-dimensional base-manifold $K_{6}$. The shape of the warp-factor $e^{2 \alpha}$ will depend on the detailed geometry of the CY four-fold $K_{8}$, as well as on other data such as the possible non-zero expectation values of other fields and the locations of the possible D-branes. 
Besides the ten-dimensional space-time metric, the fields that can take non-trivial expectation values are the following:

(i) the dilaton field

(ii) the RR-scalar or axion field

$\tilde{\phi}$

(iii) the NSNS 3-form field strength

$H^{N S}$

(iv) the RR 3-form field strength

$H^{R}$

(v) the RR 5-form field strength $F^{(5)}$

The expectation values of all these fields can all be conveniently characterized in terms of the geometry of $K_{8}$.

In the type IIB theory, the modulus $\tau$ of the elliptic fibration, the shape of the twotorus inside the $K_{8}$, describes the variation along the 6 -d base manifold $K_{6}$ of the dilaton and axion fields, $\phi$ and $\widetilde{\phi}$, via the identification

$$
\tau=\tilde{\phi}+i e^{-\phi}
$$

As mentioned above, a key feature of F-theory is that this modulus in general has nontrivial monodromies around 4-d submanifolds inside $K_{6}$. These 4-d sub-manifolds are associated with the locations of D7-branes, of which the remaining 3+1-dimensions span the uncompactified space-time directions. In going around one of the D7-branes, the modulus field $\tau$ can pick up an $S L(2, \mathbf{Z})$ monodromy

$$
\tau \rightarrow \frac{a \tau+b}{c \tau+d}
$$

which leaves the geometric shape of the two-torus fibre inside $K_{8}$ invariant, but nonetheless via (3) amounts to a non-trivial duality transformation of the IIB string theory. We thus notice that the dilaton and axion field are not smooth single-valued functions, but instead are multi-valued with branch cut singularities at the locations of the D7-branes. The full non-perturbative string theory, however, is expected to be well-behaved everywhere.

For the following, it will be convenient to combine the NSNS and RR three-from field strengths, $H^{N S}$ and $H^{R}$, of the IIB supergravity into a single four-form field-strength $G$ on $K_{8}$ as follows [9]. Let $z$ and $\bar{z}$ denote the coordinates along the $T^{2}$ fiber. Then we can write

$$
\begin{gathered}
G=\frac{\pi}{i \tau_{2}}(H \wedge d \bar{z}-\bar{H} \wedge d z) \\
H=H^{R}-\tau H^{N S} ; \quad \bar{H}=H^{R}-\bar{\tau} H^{N S}
\end{gathered}
$$


For supersymmetric configurations, $H$ defines an integral harmonic (1,2)-form on $K_{6}$ satisfying $H \wedge k=0$ with $k$ the Kähler class of $K_{6}$ [9]. It transforms under the $S L(2, Z)$ monodromy transformations (4) around the seven-branes as $H \rightarrow H /(c \tau+d)$. The fieldstrength $G$ is invariant under these transformations.

An important aspect of F-theory compactifications is that they typically carry, via their non-trivial topology, an effective total D3-brane charge. The value of this charge is proportional to the Euler characteristic $\chi\left(K_{8}\right)$ of the original Calabi-Yau four-fold $K_{8}$. Here $\chi\left(K_{8}\right)$ is defined via

$$
\frac{1}{24} \chi\left(K_{8}\right)=\int_{K_{8}} I_{8}(R)
$$

where

$$
I_{8}(R)=\frac{1}{192}\left(\operatorname{tr} R^{4}-\frac{1}{4}\left(\operatorname{tr} R^{2}\right)^{2}\right)
$$

with $R$ the curvature two-form on $K_{8}$. Global tadpole cancellation, or conservation of the RR 5-form flux, requires that this charge must be canceled by other sources. These other sources come from possible non-zero fluxes of the NSNS or RR two-form fields, or from the explicit insertion of $N$ D3-brane world branes, that is, D3-branes that span the 3+1-d uncompactified world but are localized as point-like objects inside the $K_{6}$. The number of such D3-branes is therefore not free, but completely determined via charge conservation. This global tadpole cancellation relation reads

$$
N=\frac{1}{24} \chi\left(K_{8}\right)-\frac{1}{8 \pi^{2}} \int_{K_{8}} G \wedge G
$$

Depending on the topology of $K_{8}, N$ can reach values of up to $10^{3}$ or larger. An example with $N=972$, mentioned in [8], is provided by an elliptically fibered CY four-fold over $\mathbf{P}^{3}$. The Euler number $\chi\left(K_{8}\right)$ can be non-zero only if $K_{6}$ has a non-vanishing first Chern class, that is, provided the F-theory compactification makes use of a non-zero number of D7-branes.

The equation of motion for the warp factor $e^{2 \alpha}$ obtained in [5] and [6] reads as follows

$$
\Delta^{(8)} e^{-4 \alpha}=* 4 \pi^{2}\left\{I_{8}(R)-\frac{1}{8 \pi^{2}} G \wedge G-\sum_{i=1}^{N} \delta^{(8)}\left(y-y_{i}\right)\right\}
$$

where $\Delta^{(8)}$ denotes the Laplacian and $*$ the Hodge star on $K_{8}$. The points $y=y_{i}$ correspond to the location of the $N$ D3-branes. Here, following [6], we have written the 


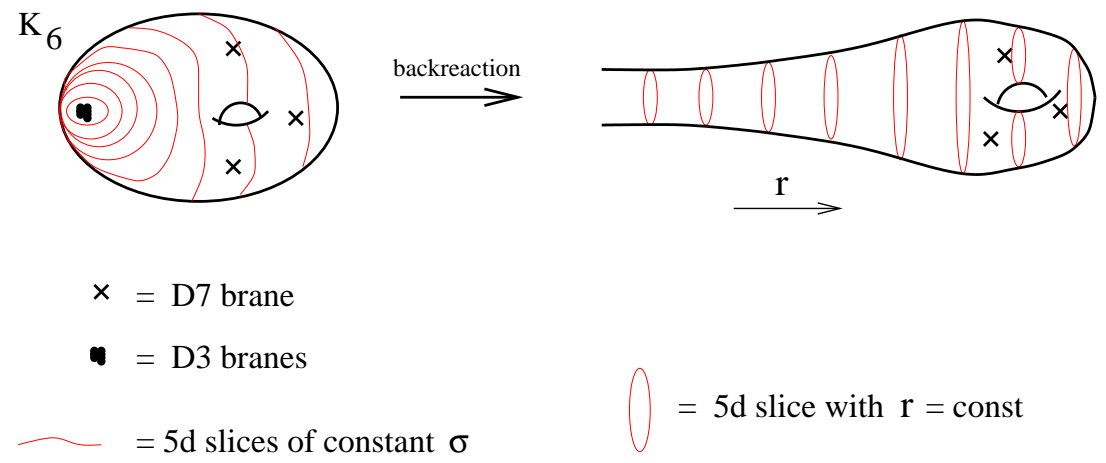

Fig 2. The contours with constant warp factor $e^{2 \sigma}$ define a particular slicing of the 6 dimensional compactification manifold $K_{6}$, which can be used to represent $K_{6}$ as a one parameter flow along $r$ of five-manifolds $K_{5}$. Upon dimensional reduction to 5 dimension, this geometry describes a one-sided RS-domain wall solution.

equation on the full 8-d manifold $K_{8}$, even though in F-theory the elliptic fiber $T^{2}$ inside $K_{8}$ has been shrunk to zero size. In this limit, the solution for $\alpha$ obtained via (10) only depends on the 6 coordinates $y^{\mathrm{M}}$ on $K_{6}$. Alternatively, using the analysis of [《], one may also first reduce the right-hand side to $K_{6}$, via integration over the $T^{2}$ fiber, and then solve the reduced equation to obtain the function $e^{-4 \alpha}$ directly on $K_{6}$.

Finally, there is also an non-trivial expectation value for the self-dual RR five-form field strength, equal to [5] [6]

$$
F_{\mu \nu \lambda \sigma \mathrm{M}}=\epsilon_{\mu \nu \lambda \sigma} \partial_{\mathrm{M}} e^{-4 \alpha} .
$$

We note that via (10) the D3-branes indeed form a source for this field strength, but that via (9) the total charge adds up to zero.

\section{Shape of the warp factor}

Let us summarize. Starting from the elliptically fibered CY four-fold $K_{8}$ we can extract a complete characterization of the warped compactification. First, since $K_{8} \simeq K_{6} \times T^{2}$, we obtain the metric $g_{\mathrm{MN}}$ on the base $K_{6}$, as well as the dilaton and axion via (3). We then deduce the form of the warp factor $e^{2 \alpha}$ from (10), which incorporates the complete backreaction due to the $G$-flux and D3-branes. Finally from (2), we obtain the actual compactification geometry. Note that, as indicated in fig 2 , the rescaling by $e^{-2 \alpha}$ of $g_{\mathrm{MN}}$ in (2) may have a drastic effect on the shape of the compactification manifold, which indeed 
may look quite different from that of the original $K_{6}$. In particular, it is possible that near the locations of the D3-branes one of the internal directions may become non-compact.

We may formally solve the equation (10) via

$$
e^{-4 \alpha(y)}=e^{-4 \alpha_{0}}+4 \pi^{2} \int d^{8} y^{\prime} \sqrt{g} \mathcal{G}\left(y, y^{\prime}\right)\left[I_{8}\left(R\left(y^{\prime}\right)\right)-\frac{1}{8 \pi^{2}} G \wedge G-\sum_{i=1}^{N} \delta^{(8)}\left(y^{\prime}-y_{i}\right)\right]
$$

where $\mathcal{G}\left(y, y^{\prime}\right)$ denotes the Green function for $\Delta^{(8)}$. The term $e^{-4 \alpha_{0}}$ parametrizes the constant zero mode of $e^{-4 \alpha}$, which is not fixed by eqn (10). Note that for $e^{-4 \alpha}$ to be everywhere positive, this constant $e^{-4 \alpha_{0}}$ can not be arbitrarily small, since the second term on the r.h.s. of (12) can become negative. This implies that the warped 6-geometry automatically has a minimal volume?.

An interesting limiting case is when all D3 branes are concentrated in one point, say $y=y_{0}$. Close to this point, the warp function $\alpha(y)$ reduces to

$$
\alpha(y) \simeq \log \left|y-y_{0}\right|+\text { const. }
$$

Via (2) this describes the familiar semi-infinite near-horizon geometry of $N$ D3-branes: $A d S_{5} \times S^{5}$ with radius $R=\sqrt[4]{4 \pi N g_{s}}$. (See fig 2.) Although the radial $A d S_{5}$ coordinate $r \simeq-R \log \left|y-y_{0}\right|$ runs over semi-infinite range, the compactification geometry (2) still gives rise to a 4 -d Einstein action with a finite 4 -d Newton constant $1 / \ell_{4}^{2}$ equal to

$$
\frac{1}{\left(\ell_{4}\right)^{2}}=\frac{1}{\left(\ell_{10}\right)^{8}} \int_{K_{6}} \sqrt{g} e^{2 \phi-4 \alpha}
$$

with $\ell_{10}$ the $10-d$ Planck length.

\section{Reduction to 5 dimensions.}

We would now like to show that these F-theory compactifications, upon performing a suitable dimensional reduction to five dimensions, reduce to supersymmetric RS domain wall solutions. To this end we will look for a specific coordinate system

$$
y^{\mathrm{M}}=\left(y^{m}, r\right)
$$

where $m$ now runs over 5 values, such that the 10 -d metric takes the following form

$$
d s_{R S}^{2}=e^{2 \sigma(r)} \eta_{\mu \nu} d x^{\mu} d x^{\nu}+d r^{2}+h_{m n}(y, r) d y^{m} d y^{n}
$$

\footnotetext{
${ }^{2}$ We thank Sav Sethi for bringing this feature to our attention.
} 
where $d s_{R S}^{2}$ related to the original IIB string metric (2) via the rescaling

$$
d s_{R S}^{2}=e^{\phi / 2}\left(V_{5}\right)^{1 / 4} d s_{I I B}^{2} \quad V_{5}=\frac{1}{\left(\ell_{10}\right)^{5}} \int_{K_{5}} \sqrt{h}
$$

Here the prefactor in (17) is chosen such that $d s_{R S}^{2}$ is the metric in the 5-d Einstein frame (where we have taken the 5 -d Planck length $\ell_{5}$ equal to the $10-\mathrm{d}$ one). The 5 - $\mathrm{d}$ slices of constant $r$ define 5 -d submanifolds $K_{5}$, on which $2 \alpha(y)+\phi(y) / 2=$ constant; this correspondence guarantees that the warp-factor $e^{2 \sigma}$ in $d s_{R S}^{2}$ just depends on $r$ and not on the remaining $y^{m}$ 's.

In this way we indeed obtain a solution that from the 5-d perspective looks just like an RS-type warped geometry. For large negative $r$, close to the D3-branes, the warp factor behaves like $e^{2 \sigma} \simeq e^{-2|r| / R}$ with $R$ the AdS-radius of the $N$ D3-brane solution. On the other end, somewhere outside the throat region of the AdS-tube, near the 'equator' of the $K_{6}$, the warp factor $e^{2 \sigma(r)}$ reaches some maximal value. Eventually, there is a boundary value for the coordinate $r$, which we can take to be $r=0$, at which the transverse 5manifold $K_{5}$ shrinks to zero size. However, as is clear from fig 2 , this does not correspond to any singularity, but just to a smooth cap closing off the 6 -d manifold $K_{6}$. This fact that $r$ takes a maximal value $r=0$ implies that the AdS-space is indeed compactified, in the sense that, relative to the 4 - $d$ space-time, it has a finite volume. It therefore produces to a finite 4-d Newton constant equal to (cf [1] )

$$
\frac{1}{\left(\ell_{4}\right)^{2}}=\frac{1}{\left(\ell_{5}\right)^{3}} \int_{-\infty}^{0} d r e^{2 \sigma(r)}
$$

It is easily verified that via (17) and (16), this result coincides with the value (14) found via direct reduction from 10 dimensions.

Upon dimensional reduction, the metric $h_{m n}$ of the internal $K_{5}$, as well as other fields such as the dilaton, all reduce to scalar fields that provide the matter multiplets of the 5 - $d$ supergravity. All these fields vary with the radial coordinate $r$, and since this radial flow is supersymmetric, it should in principle be described as some gradient flow driven by some appropriate superpotential. However, due to the rather complex geometrical structure of the typical F-theory compactification, it unfortunately seems impossibly hard to find the explicit form of this potential. 


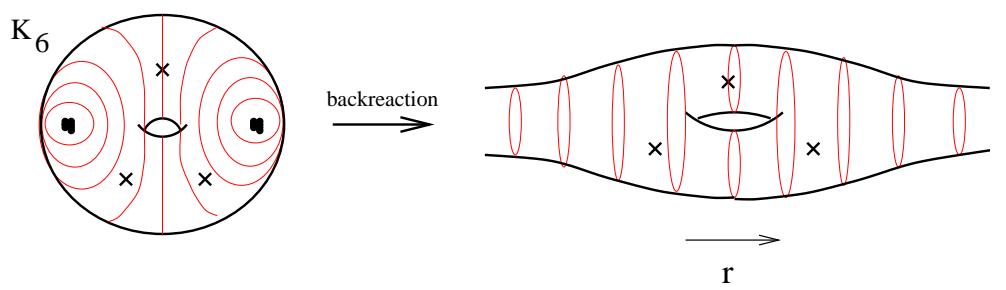

Fig 3. An RS domain wall in between two AdS-type regions can be obtained by starting with a $\mathbf{Z}_{2}$ symmetric 6-manifold $K_{6}$, in which the D3-branes are located at opposite image points under the $\mathbf{Z}_{2}$.

\section{Discussion}

In this note we have summarized the geometrical description of warped F-theory compactifications, and shown they can be used to obtain geometries very analogous to the RS-scenario. Due to the presence of the D7-branes in the F-theory geometry, the solutions are not completely smooth: the dilaton and axion field have isolated branch cut singularities at the D7-brane loci around which $\tau$ is multi-valued. The string theory, however, is well-defined in this background.

Our description of the solutions makes clear that the internal structure of the RS Planck brane is not that of an actual brane, but rather that of a compactification geometry (which however also contains the D7-branes). Consequently, the localized graviton zero mode of [1] is just the standard KK zero mode of the 10-d metric; because its wavefunction is sharply peaked near the wall region, where the warp factor $e^{2 \sigma}$ is maximal, the 4-d graviton indeed looks like some bound state. From the higher dimensional viewpoint, however, this RS-localization of gravity is not a new phenomenon.

The most interesting aspect of these warped string compactifications, is that there is no clear distinction between the low energy and extra dimensional physics. Kaluza-Klein excitations, when localized far inside the AdS region can describe particles with masses much smaller than the inverse size of the original $K_{6}$. While these particles naively look like new degrees of freedom arising from the presence of extra dimensions, the holographic AdS/CFT correspondence [11] tells us that they are in fact localized excitations of the low energy gauge theory. The same holds for string excited modes in this region. Hence via the holographic identification of the $R G$ scale with a real extra dimension, the two usually separate stages of dimensional and low energy reduction should now be combined into one single procedure.

Finally, as a closely related point, we need to emphasize that the solutions as given 
here are generically unstable against small perturbations. The best way to understand this instability is via the RG language: in general there will exist relevant operators whose couplings, once turned on in the UV region, will quickly grow and typically produce some singularity that effectively closes off the AdS-tube [12]. In our way of obtaining the solutions, we did not immediately notice this instability, because we required that the original $K_{6}$ geometry and all other fields, except for the warp-factor and the 5-form flux, are smooth at the locations of the D3-branes. This requirement is special, however, and we should allow for deformations that may spoil this property.

In order to ensure that the perturbed geometry contains a substantial intermediate AdS-like region, we either need to fine-tune the UV initial conditions or introduce a symmetry that eliminates these unstable modes. In RG language, this means that the dual 4-d field theory should be made approximately conformal invariant over a large range of scales, separating the Planck scale from the scale set by the non-trivial gauge dynamics.

Via the AdS/CFT dictionary, the problem of realizing an RS-geometry in string theory therefore is reduced back to the original problem it was designed to solve, namely how to generate a large gauge hierarchy. Or stated in more positive terms, in searching for realistic string compactification scenarios, the observed gauge hierarchy can be viewed as an indication that warped geometries of this type deserve serious attention.

\section{ACKNOWLEDGEMENTS}

This work is supported by NSF-grant 98-02484. C.S.C is supported in part by an NSF Graduate Research Fellowship. We would like to thank M. Berkooz, M. Cvetic, R. Dijkgraaf, M. Gremm, R. Kallosh, L. Randall, S. Sethi and E. Verlinde for helpful discussions.

\section{References}

[1] L. Randall and R. Sundrum, A Large Mass Hierarchy from a Small Extra Dimension, Phys. Rev. Lett. 83 (1999) 3370, hep-ph/9905221. An Alternative to Compactification, Phys. Rev. Lett. 83 (1999) 4690, hep-th/9906064.

[2] R. Kallosh and A. Linde, Supersymmetry and the Brane World, hep-th/0001071

[3] M. Cvetic, H. Lu, C.N. Pope, Localised Gravity in the Singular Domain Wall Background?, hep-th/0002054.

[4] H. Verlinde, Holography and Compactification, hep-th/990 6182 
[5] K. Becker and M. Becker, "M-Theory on Eight-Manifolds" Nucl.Phys. B477 (1996) hep-th/9605053

[6] K. Dasgupta, G. Rajesh and S. Sethi, "M Theory, Orientifolds and G-Flux" JHEP 9908 (1999) hep-th/9908088

[7] E. Witten, "Phase Transitions In M-Theory And F-Theory," Nucl.Phys. B471 (1996) 195-216, hep-th/9603150.

[8] S. Sethi, C. Vafa and E. Witten, "Constraints on Low-Dimensional String Compactifications" Nucl.Phys. B480 (1996) 213-224 hep-th/9606122

[9] S. Gukov, C. Vafa and E. Witten, "CFT's From Calabi-Yau Four-folds" hepth/9906070

[10] C. Vafa, "Evidence for F-Theory," hep-th/9602022

[11] J. Maldacena, Adv. Theor. Math. Phys. 2 (1998) 231, hep-th/9711200.

[12] J. Polchinski and M. Strassler, "The String Dual of a Confining Four-Dimensional Gauge Theory," hep-th/0003136 